\title{
ARTICLE
}

Clinical Study

\section{SCOT: a comparison of cost-effectiveness from a large randomised phase III trial of two durations of adjuvant Oxaliplatin combination chemotherapy for colorectal cancer}

\author{
José Robles-Zurita ${ }^{1}$, Kathleen A. Boyd ${ }^{1}$, Andrew H. Briggs ${ }^{1}$, Timothy Iveson ${ }^{2}$, Rachel S. Kerr ${ }^{3}$, Mark P. Saunders ${ }^{4}$, Jim Cassidy ${ }^{5}$, \\ Niels Henrik Hollander ${ }^{6}$, Josep Tabernero ${ }^{7}$, Eva Segelov ${ }^{8}$, Bengt Glimelius ${ }^{9}$, Andrea Harkin ${ }^{5}$, Karen Allan ${ }^{5}$, John McQueen ${ }^{5}$, \\ Sarah Pearson ${ }^{10}$, Ashita Waterston ${ }^{11}$, Louise Medley ${ }^{12}$, Charles Wilson ${ }^{13}$, Richard Ellis ${ }^{14}$, Sharadah Essapen ${ }^{15}$, Amandeep S. Dhadda ${ }^{16}$, \\ Rob Hughes ${ }^{17}$, Stephen Falk ${ }^{18}$, Sherif Raouf ${ }^{19}$, Charlotte Rees ${ }^{2}$, Rene K Olesen ${ }^{6}$, David Propper ${ }^{20}$, John Bridgewater ${ }^{21}$, Ashraf Azzabi ${ }^{22}$, \\ David Farrugia $^{23}$, Andrew Webb ${ }^{24}$, David Cunningham ${ }^{25}$, Tamas Hickish ${ }^{26}$, Andrew Weaver ${ }^{27}$, Simon Gollins ${ }^{28}$, Harpreet S Wasan ${ }^{29}$ and \\ James Paul ${ }^{5}$
}

BACKGROUND: The Short Course Oncology Therapy (SCOT) study is an international, multicentre, non-inferiority randomised controlled trial assessing the efficacy, toxicity, and cost-effectiveness of 3 months $(3 \mathrm{M})$ versus the usually given 6 months (6 M) of adjuvant chemotherapy in colorectal cancer.

METHODS: In total, 6088 patients with fully resected high-risk stage II or stage III colorectal cancer were randomised and followed up for 3-8 years. The within-trial cost-effectiveness analysis from a UK health-care perspective is presented using the resource use data, quality of life (EQ-5D-3L), time on treatment (ToT), disease-free survival after treatment (DFS) and overall survival (OS) data. Quality-adjusted partitioned survival analysis and Kaplan-Meier Sample Average Estimator estimated QALYs and costs. Probabilistic sensitivity and subgroup analysis was undertaken.

RESULTS: The $3 \mathrm{M}$ arm is less costly $(-£ 4881 ; 95 \% \mathrm{Cl}$ : $-£ 6269 ;-£ 3492)$ and entails (non-significant) QALY gains $(0.08 ; 95 \% \mathrm{Cl}$ : -0.086 ; 0.230 ) due to a better significant quality of life. The net monetary benefit was significantly higher in $3 \mathrm{M}$ under a wide range of monetary values of a QALY. The subgroup analysis found similar results for patients in the CAPOX regimen. However, for the FOLFOX regimen, $3 \mathrm{M}$ had lower QALYs than $6 \mathrm{M}$ (not statistically significant).

CONCLUSIONS: Overall, $3 \mathrm{M}$ dominates $6 \mathrm{M}$ with no significant detrimental impact on QALYs. The results provide the economic case that a $3 \mathrm{M}$ treatment strategy should be considered a new standard of care.

British Journal of Cancer (2018) 119:1332-1338; https://doi.org/10.1038/s41416-018-0319-z

\section{INTRODUCTION}

Colorectal cancer (CRC) affects 1.36 million patients worldwide each year, and in the UK, is the fourth most common malignancy, accounting for $12 \%$ of all new cancer cases each year- 41,265 in 2014. ${ }^{1}$ The cost of treatment for CRC within the first year of diagnosis is considerably larger than that for treating other common cancers such as breast, lung and prostate, and it is estimated that CRC costed the English health-care system $£ 542$ million in $2010 .^{2}$ Many authors have also commented on the rising cost of cancer care $^{3}$ and the challenge this presents for high income countries to provide care to increasing populations of cancer patients.

Given recent budget restrictions and rising pressure on healthcare systems around the world, there is a need to ensure that cancer treatments offer the best value for money and provide policy makers with up-to-date information on both effectiveness and cost-effectiveness to help inform efficient allocation of healthcare resources. In the treatment of CRC, adjuvant chemotherapy is

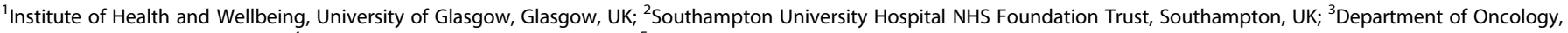

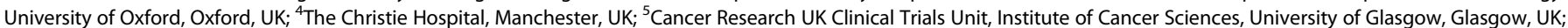

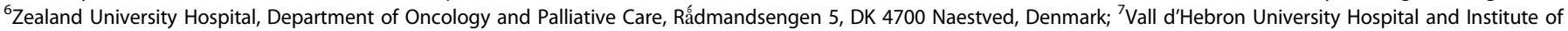

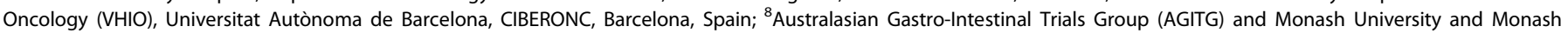

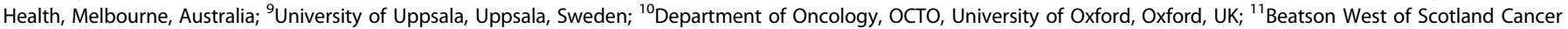

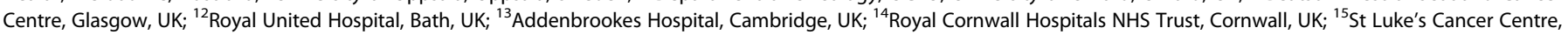

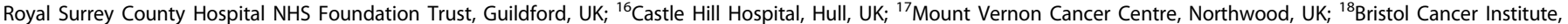

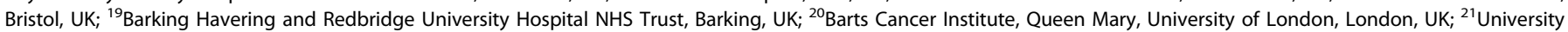

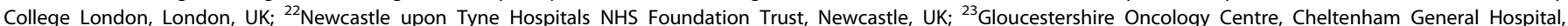

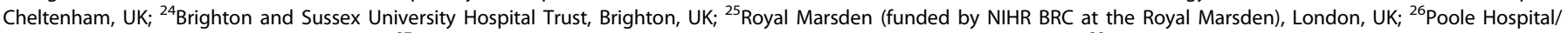

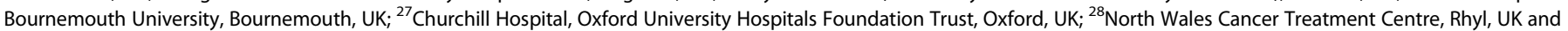
${ }^{29}$ Hammersmith Hospital, Imperial College London, London, UK

Correspondence: José. Robles-Zurita (JoseAntonio.Robles-Zurita@glasgow.ac.uk)

Received: 6 April 2018 Revised: 3 October 2018 Accepted: 9 October 2018

Published online: 13 November 2018 
more effective than surgery alone for patients with fully resected CRC at stage $\mathrm{III}^{4-11}$ and, to a lesser extent, at high-risk stage II. ${ }^{12-14}$ However, the current 6 month standard duration of adjuvant chemotherapy is associated with considerable side effects ${ }^{15,16}$, which impact on patient quality of life. It has previously been unknown whether 3 months of adjuvant chemotherapy treatment duration could reduce these side effects with no detrimental impact on efficacy. Given the escalating cost of chemotherapy treatments of $\mathrm{CRC}^{17}$, it is pertinent that the cost-effectiveness of alternative treatment durations and regimens ${ }^{18,19}$ is explored.

This paper reports on an economic evaluation undertaken alongside the Short Course Oncology Trial (SCOT) randomised controlled trial (ISRCTN No: 23516549) to explore the costeffectiveness of 3 month (3M) versus 6 months (6 M) adjuvant chemotherapy treatment in stage III/high-risk stage II CRC patients.

\section{METHODS}

The SCOT trial (ISRCTN No.: 23516549) was an international phase III randomised controlled trial, to assess non-inferiority of $3 \mathrm{M}$ versus $6 \mathrm{M}$ of oxaliplatin/FP adjuvant chemotherapy in stage III/ high-risk stage II CRC patients. Patients were randomised to receive either 3 or 6 months of oxaliplatin-containing adjuvant treatment. The chemotherapy regimen (FOLFOX or CAPOX) was at the choice of the patient and/or physician prior to randomisation. The trial recruited 6088 patients across six countries (UK, Denmark, Spain, Sweden, Australia and New Zealand), followed up for a minimum of 3 years, up to 8 years post randomisation. SCOT was designed as a non-inferiority trial, aiming to exclude a maximum $2.5 \%$ fall in in 3-year disease-free survival from halving the adjuvant treatment duration. Secondary endpoints were overall survival, toxicity, quality of life and cost-effectiveness. Further details of the intervention, randomisation, methods and outcomes for the SCOT trial are reported elsewhere. ${ }^{20}$

The aim of the economic evaluation is to explore the treatment and hospitalisations costs, quality of life and survival outcomes of $3 \mathrm{M}$ versus $6 \mathrm{M}$ adjuvant chemotherapy treatment within the timeframe of the SCOT clinical trial.

The cost-effectiveness analysis was undertaken from the perspective of the UK NHS and Personal Social Services for the 2016 base year, adhering to good practice guidelines. ${ }^{21,22} \mathrm{~A}$ within-trial analysis utilised the individual patient-level data on resource use, quality of life (EQ-5D-3L) and survival. The cost (C) and quality-adjusted life year (QALY) outcomes for each arm are estimated and combined with the UK decision threshold for costeffectiveness $(\lambda)$ of $£ 30,000 / Q A L Y^{21}$ to report outcomes in terms of Net Monetary Benefit (NMB), according to good reporting practice guidance. $^{23}$ (NMB is calculated by monetarising the measure of effectiveness (QALYs) by explicitly incorporating the decision threshold $(\lambda)$, and then subtracting the cost $(C)$ to determine whether the NMB is greater than zero. Specifically: NMB $=\mathrm{QALY} \times$ $\lambda-C$. The strategy with the greatest NMB is the cost-effective choice.) The incremental NMB is the difference between the NMB of the two arms.

\section{Outcomes}

The effectiveness measure for the economic analysis is the discounted QALY gains per patient in each of the study arms. QALYs are calculated using quality-adjusted survival analysis. ${ }^{24}$ Overall survival is partitioned into three different health states: time on treatment (ToT), disease-free survival after treatment (DFS) and recurrence. The Kaplan-Meier survival estimates are used for the computation of the quality-adjusted survival time in each health state over the 8 year within-trial period.

A separate model estimated quality of life for each health state. EQ-5D-3L data collected for a subsample of 1832 patients (about $30 \%$ of the study sample), at baseline and follow-up, and combined with the UK EQ-5D-3L health utility scores ${ }^{25}$ to calculate utilities. A linear regression with standard errors clustered at the individual level estimated quality of life including independent variables: health states, treatment group and individual characteristics.

Time in the health states ToT, DFS and recurrence was computed by integration of the Kaplan-Meier curves and then adjusted by quality of life using the method of integrated qualitysurvival product ${ }^{24}$ to compute QALYs. This approach to qualityadjusted survival analysis avoids problems of informative censoring in survival analysis based on individual QALYs as an endpoint. $^{26}$

Costs

Costs were calculated by the measurement and valuation of resources used by the SCOT trial participants during the treatment and follow-up periods. The trial collected patient-level resource use data on adjuvant chemotherapy doses and duration and hospitalisations during treatment and follow-up for the whole study sample. All costs have been valued in 2016 pounds sterling.

Adjuvant chemotherapy: The doses of Oxaliplatin, Capecitabine, 5 -fluouracil bolus injection and 5-fluorouracil continuous infusion were collected and combined with their respective unit costs. The cost per $\mathrm{mg}$ of each drug is obtained from the British National Formulary $73^{27}$ as detailed in Table S1 in the supplementary material.

Hospitalisation: Hospitalisation costs are incurred by patients for receiving adjuvant chemotherapy during the treatment period and also for treating adverse reactions. Hospitalisation resource use data include night stays in intensive care unit (ICU), high dependency unit (HDU), general medicine, and in-patient chemotherapy (IP) as well as day cases (DC) and outpatient attendances (OP). The direct and non-direct costs for each hospitalisation were obtained from the Information Services Division (ISD) ${ }^{28}$ of the National Health Service Scotland. Direct costs can be classified by medical, nursing, health professionals, pharmacy, theatre, laboratory and others. For IPs and DCs occurring within the treatment period, the pharmacy cost is subtracted to avoid double counting of chemotherapy medication. Table S2 in the supplementary material details unit costs for each type of hospitalisation. These costs include staff administration time, e.g., for delivering the adjuvant chemotherapy, in medical and nursing costs and non-direct costs when a hospitalisation or appointment for chemotherapy has occurred. The cost of treating adverse events is assumed to be included in hospitalisation costs for patients attending hospital for night, day case or outpatient visit after having adverse reaction.

Kaplan-Meier Sample Average (KMSA) Estimator: Given that the follow-up period differed among patients total cost per patient is estimated by the KMSA estimator. ${ }^{29}$ This way the average total cost is estimated as the sum of the average cost for each period multiplied by the probability of surviving at the beginning of the period.

\section{Cost-effectiveness outcome}

The analyses were performed using STATA 14.0 (StataCorp, TX, USA) to compare the mean costs, and mean QALY differences between the comparator groups (3 months vs. 6 months treatment) and the NMB is reported in line with recent reporting guidelines ${ }^{23}$ and the UK reference case. ${ }^{21}$ Discounting of costs and QALY outcomes beyond 1 year was applied at $3.5 \%$ rate as recommended. ${ }^{21}$

\section{Missing data}

Only a subgroup of patients reported EQ-5D health status following recommendations to discontinue data collection based on an interim study analysis. ${ }^{30}$ To control for plausible differences between the EQ-5D sample and the total study sample the quality 
of life model includes co-variables such as planned treatment, high-risk disease (T4 or N2 as previously defined ${ }^{20}$ ), gender, age and ethnicity. The model predicts health utilities for the average characteristics of the patients in each health state.

\section{Sensitivity analysis}

Bootstrapping (1000 iterations) ${ }^{31}$ was used to account for uncertainty around the difference in costs and QALYs and how this uncertainty impacts on the cost-effectiveness outcome. The uncertainty is reported through confidence intervals and the computation of cost-effectiveness acceptability probabilities estimated as a function of the threshold for the monetary value of a QALY.2

Subgroup analyses was undertaken, in line with the main trial analysis, ${ }^{20}$ to consider cost-effectiveness of the two treatment duration strategies according to: planned treatment regimen, CAPOX/FOLFOX; disease risk, high/low risk stage III; gender; and age.

\section{RESULTS}

Health outcomes

In total, 6088 patients were randomised in the SCOT study, however, only 6065 patients are considered in the analysis since 23 patients withdrew consent to use their data at follow-up. ${ }^{20}$ Table 1 illustrates how overall expected survival time, over 8 years after randomisation, is split into ToT, DFS and recurrence. ToT is significantly higher in the $6 \mathrm{M}$ arm while DFS just fails to significantly favour the $3 \mathrm{M}$ arm at $5 \%$ of error. No statistically significant differences are found for time in recurrence or overall survival. Figure 1 shows the area representing ToT, DFS and recurrence time generated from Kaplan-Meier estimates (see more detailed Kaplan-Meier curves in supplementary material: Figures S1, S2 and S3).

Quality of life

Table 2 shows the results of the utility model for non-missing observations. The effect of recurrence and time on treatment is capture by two indicators taking value 1 for all those EQ-5D responses occurring in those health states. ToT and recurrence have a significant negative effect on utility, as could be expected. The $3 \mathrm{M}$ arm is estimated to have higher quality of life $(P$-value $<$ 0.05 ) even after controlling for recurrence and time on treatment. These results are consistent with the higher incidence of adverse events in the $6 \mathrm{M}$ groups compared with the $3 \mathrm{M}$ arm. For example, the main long-term adverse event was neuropathy associated with the Oxaliplatin component of the chemotherapy regimen. Specifically, $58 \%$ of patients in the $6 \mathrm{M}$ arm had peripheral neuropathy equal or worse than Grade 2, the same

Table 1. Overall survival time by health state (ToT, DFS and recurrence) and by arm

\begin{tabular}{lllll}
\hline & $3 \mathrm{M}$ & $6 \mathrm{M}$ & \multicolumn{2}{l}{ Incremental } \\
\hline Survival analysis & Mean & Mean & Mean & $P$-value \\
$N$ & 3035 & 3030 & 6065 & \\
ToT & 0.21 & 0.39 & -0.18 & 0.000 \\
DFS & 5.93 & 5.74 & 0.19 & 0.053 \\
Recurrence & 0.73 & 0.77 & -0.041 & 0.605 \\
Total (OS) & 6.87 & 6.90 & -0.032 & 0.695 \\
\hline
\end{tabular}

Kaplan-Meier estimates used for computation of expected time in each health state

Survival time estimated up to 8 years post randomisation

Estimation sample in the case of ToT is lower due to missing values, 3018 and 3013 patients for the $3 \mathrm{M}$ and $6 \mathrm{M}$ arms, respectively figure for the $3 \mathrm{M}$ arm was $25 \%$. Patients' characteristics are included in the model to adjust health utilities to the average values of the whole SCOT sample, with and without EQ-5D data (see Table S3 in supplementary material for differences in characteristics between subsamples). Significant characteristics are male and age, with a positive effect, and ethnicity, with a lower quality of life for African/Caribbean patients and South Asian with respect to White/Caucasian. No significant variables are planned treatment and disease risk. The results of the model follow the pattern of the evolution of EQ-5D scores over time for both arms in Fig. 2. Figures S1-S5 From randomisation point quality of life decreases for both arms up to 3 months. At this point health utilities for those in $3 \mathrm{M}$ arm increases as they finish treatment while those patients in the $6 \mathrm{M}$ arm continue with lower quality of life. Changes in quality of life are related to time on treatment although differences between the two arms remain after receiving adjuvant chemotherapy to some extent.

\section{Costs}

Table 3 details cost incurred by patients in both arms, with a breakdown of the hospitalisation costs over different time periods. As expected, adjuvant chemotherapy costs are higher in the $6 \mathrm{M}$ arm. Hospitalisation costs differ between arms in the 4-6-month period, as could be expected; however, the data also reveal that patients in the $6 \mathrm{M}$ arm experience more hospitalisation costs in the 7-12-month period, possibly reflecting longer lasting complications due to the longer treatment period. Interestingly, the costs during the first 6 months after randomisation are not doubled for the $6 \mathrm{M}$ arm. Specifically, adjuvant chemotherapy and hospitalisation costs are only 1.67 and 1.45 times higher, respectively, in the $6 \mathrm{M}$ arm. This is due to tolerability of treatment where many patients randomised to $6 \mathrm{M}$ do not complete the full course of treatment. While $83.3 \%$ of patients randomised to $3 \mathrm{M}$ received the full 3 months treatment, only $58.8 \%$ of those randomised to $6 \mathrm{M}$ received 6 months treatment. ${ }^{20}$ Notice that the health economic evaluation is clearly justified in the context of this study to analyse the actual consequences on costs from reducing the intended treatment duration from 6 to 3 months. There is no difference in cost between arms beyond 12 months. Overall, the cost is significantly greater in the $6 \mathrm{M}$ arm, driven by hospitalisations $(-£ 2835)$ in the first 6 months to a higher extent than by received adjuvant chemotherapy $(-£ 1829)$. A detailed analysis of resource use, regarding adjuvant chemotherapy and hospitalisations, can be found in Tables S4 and S5, respectively, in the supplementary material.

\section{Cost-effectiveness analysis}

Table 4 details the results of the base case cost-effectiveness analysis. The 3-month adjuvant chemotherapy strategy is significantly cheaper, costing $£ 4,881$ less than the $6 \mathrm{M}$ strategy over the 8 year analysis period. The $3 \mathrm{M}$ strategy also results in the greatest QALY gain expected, although this is not statistically significant $(P$-value $=0.33)$ and results in uncertainty in the QALY gains as seen in the $95 \%$ confidence intervals. The QALY gains for the $3 \mathrm{M}$ arm are driven by the significant improvement in quality of life rather than life expectancy (LE), indeed the $6 \mathrm{M}$ arm has a very small non-significant improvement in LE $(P$-value $=0.69)$, meeting the non-inferiority margin for survival specified for the SCOT trial. ${ }^{20}$ These cost-effectiveness results indicate that the $3 \mathrm{M}$ treatment strategy is dominant (cost saving with improvement in QALYs), with an incremental NMB of $£ 7246$ per patient. $3 \mathrm{M}$ strategy is therefore the cost-effective option, with $99 \%$ probability of being cost-effective at the UK decision threshold of $£ 30,000 / Q A L Y .{ }^{21}$ The $3 \mathrm{M}$ also remains the optimal choice across a wide range of willingness to pay values (see Figure S4 in supplementary material for the cost-effectiveness acceptability curve). 

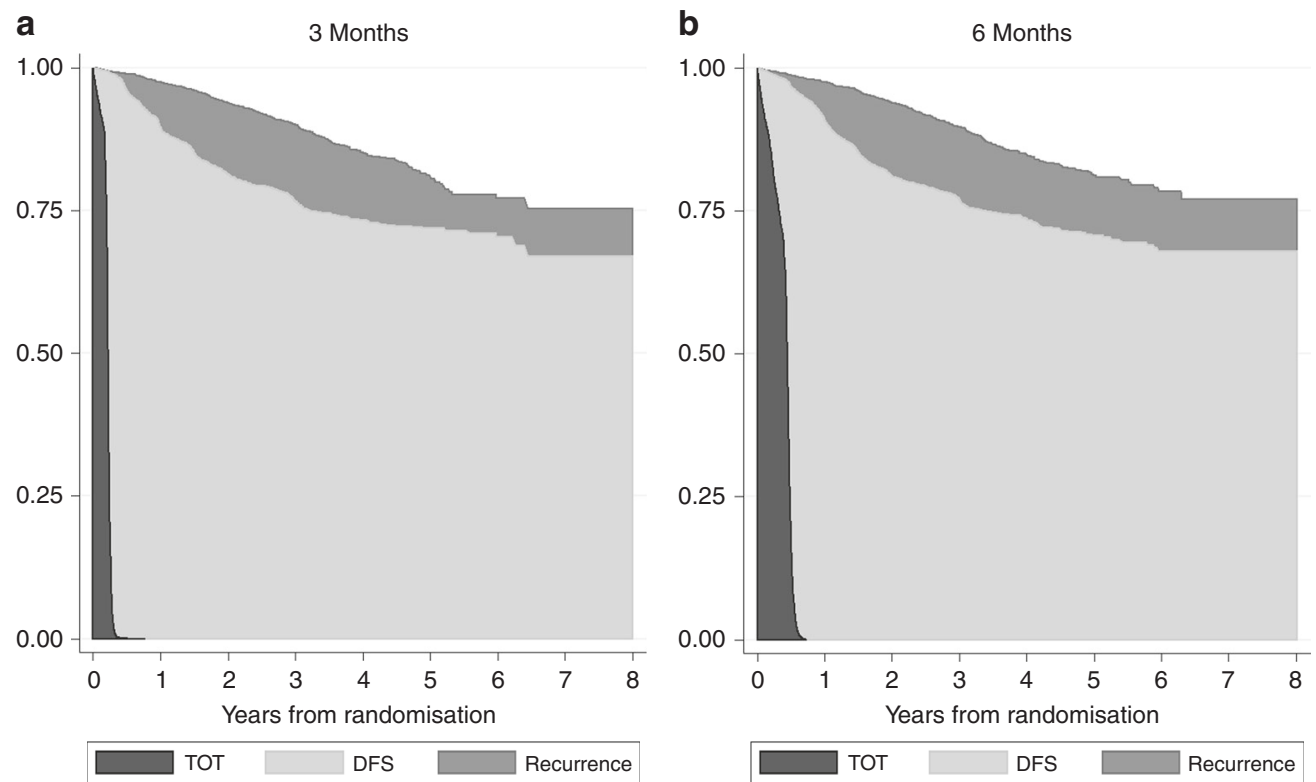

Fig. 1 Overall survival partitioned into time on treatment (ToT), disease free survival after treatment (DFS), and recurrence. Kaplan-Meier estimates over 8 years and by arms

\begin{tabular}{|c|c|c|}
\hline Variable & Coef. & S.E. \\
\hline $\mathrm{N}$-observations & 16,091 & \\
\hline $\mathrm{N}$-patients & 1757 & \\
\hline \multicolumn{3}{|c|}{ Health states (ref: disease free) } \\
\hline On treatment & $-0.0394^{* * *}$ & 0.00408 \\
\hline Recurrence & $-0.0578^{* * *}$ & 0.0139 \\
\hline Arm: 6 months & $-0.0154^{*}$ & 0.00730 \\
\hline \multicolumn{3}{|l|}{ Characteristics } \\
\hline CAPOX & 0.00402 & 0.00783 \\
\hline High risk & -0.00911 & 0.00724 \\
\hline Male & $0.0159^{*}$ & 0.00733 \\
\hline Age & $0.00162^{* * *}$ & 0.000429 \\
\hline \multicolumn{3}{|c|}{ Ethnic (ref: White/Caucasian) } \\
\hline African/Caribbean & $-0.0810^{*}$ & 0.0385 \\
\hline South Asian & $-0.145^{* *}$ & 0.0536 \\
\hline Chinese & -0.0447 & 0.0772 \\
\hline Other & 0.0178 & 0.0217 \\
\hline Constant & $0.866^{* * *}$ & 0.00944 \\
\hline \multicolumn{3}{|c|}{$\begin{array}{l}\text { Standard errors (S.E.) clustered at the patient level } \\
P \text {-values: }{ }^{*} P<0.05,{ }^{* *} P<0.01{ }^{* * *} P<0.001 \\
\text { N-observations refer to the number of EQ-5D questionnaires reported in } \\
\text { total by the } \mathrm{N} \text {-patients included in the estimation } \\
\text { The constant in the model refers to a } 65 \text {-year-old female patient, in } \\
\text { disease-free health state in the } 3 \text { months arm, receiving FOLFOX treatment, } \\
\text { with low risk stage III and White/Caucasian ethnicity }\end{array}$} \\
\hline
\end{tabular}

\section{Subgroup analysis}

Table S6 illustrates the cost-effectiveness for the subgroup analyses according to: (i) treatment regimen, (ii) risk, (iii) gender and (iv) age. For all the subgroups except FOLFOX treatment and males, the cost-effectiveness results are similar to the base case analysis with the $3 \mathrm{M}$ arm dominating and a $99 \%$ probability of being the cost-effective option. In the FOLFOX treatment group, the $3 \mathrm{M}$ arm shows cost saving, but also fewer QALY gains, which were not significant but driven by a small gain in LE for the $6 \mathrm{M}$

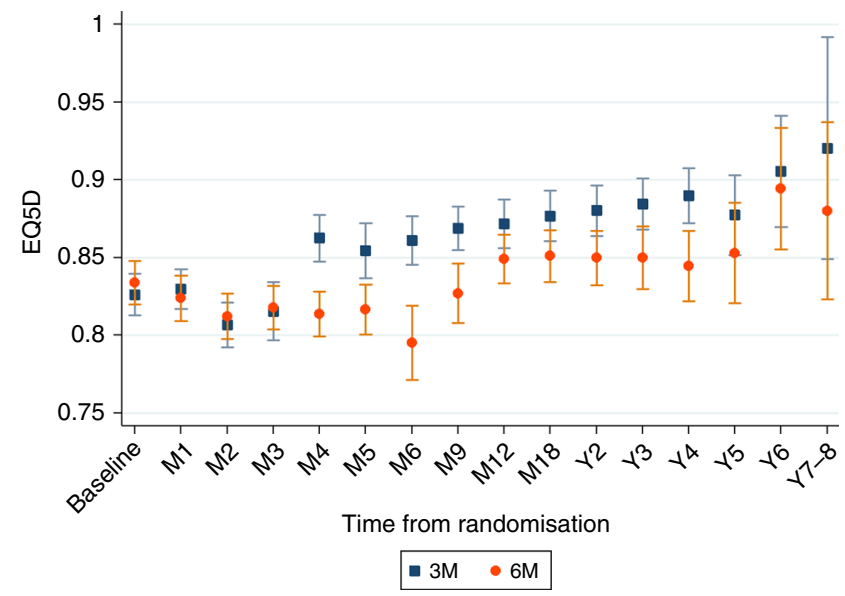

Fig. 2 Evolution of EQ-5D utilities over time by arms. Average and $95 \% \mathrm{Cl}$

Table 3. Costs by treatment duration ( $£ /$ patient $)$

\begin{tabular}{lllll}
\hline $\begin{array}{l}\text { Time from beginning of } \\
\text { treatment }\end{array}$ & $\begin{array}{l}3 \mathrm{M} \\
\text { Mean }\end{array}$ & $\begin{array}{l}6 \mathrm{M} \\
\text { Mean }\end{array}$ & $\begin{array}{l}\text { Incremental } \\
\text { Mean }\end{array}$ & $P$-value \\
\hline$N$ & 3035 & 3030 & 6065 & \\
Adjuvant chemotherapy & 2750 & 4579 & -1829 & $<0.001$ \\
Hospitalisation (0-3 months) & 3576 & 3595 & -19 & 0.816 \\
Hospitalisation (4-6 months) & 1790 & 4185 & -2395 & $<0.001$ \\
Hospitalisation (7-12 months) & 2748 & 3054 & -306 & 0.030 \\
Hospitalisation ( $>12$ months) & 8473 & 8588 & -115 & 0.876 \\
Total hospitalisation & 16587 & 19422 & -2835 & $<0.001$ \\
Total & 19337 & 24001 & -4663 & $<0.001$ \\
\hline
\end{tabular}

Figures refer to non-discounted average cost for each period conditional on survival

arm. The relative advantage of the $3 \mathrm{M}$ arm in QALYs is higher for the CAPOX treatment, high-risk patients, females and older patients. An interaction test of differences in incremental QALYs between subgroups conclude that only the treatment regimen 
Table 4. Estimates of costs and QALYs for the two strategies

\begin{tabular}{|c|c|c|c|c|c|c|c|c|c|}
\hline \multirow[t]{2}{*}{ Intervention strategies } & \multicolumn{2}{|c|}{ Costs ( $(£ /$ patient $)$} & \multicolumn{2}{|l|}{ LE } & \multicolumn{2}{|l|}{ QALYs } & \multicolumn{2}{|c|}{ NMB ( $£ 30 \mathrm{~K} / \mathrm{QALY})$} & \multirow[t]{2}{*}{ Prob CE $\lambda=£ 30,000$} \\
\hline & Mean & {$[95 \% \mathrm{Cl}]$} & Mean & {$[95 \% \mathrm{Cl}]$} & Mean & {$[95 \% \mathrm{Cl}]$} & Mean & {$[95 \% \mathrm{Cl}]$} & \\
\hline 3 Months & 18,401 & {$[17,538 ; 19,328]$} & 6.87 & {$[6.73 ; 6.99]$} & 5.30 & {$[5.17 ; 5.40]$} & 140492 & {$[135,327 ; 145,658]$} & 0.995 \\
\hline 6 Months & 23282 & {$[22,227 ; 24,367]$} & 6.90 & {$[6.78 ; 7.02]$} & 5.22 & {$[5.10 ; 5.34]$} & 133246 & {$[129,569 ; 136,922]$} & 0.005 \\
\hline Incremental & -4881 & {$[-6269 ;-3492]$} & -0.03 & {$[-0.22 ; 0.13]$} & .08 & {$[-0.086 ; 0.230]$} & 7246 & [3469; 11,023] & (3 M dominates) \\
\hline
\end{tabular}

Health utilities conditional on survival considered

KMSA estimator and partitioned survival analysis used for costs and QALYs, respectively

Cls are computed using bootstrap sampling

Probability of cost-effectiveness calculated using 1000 bootstrap replications

subgroup shows a statistically significant difference in QALY differences, CAPOX (0.19 Incremental QALYs) and FOLFOX (-0.12 Incremental QALYs) with a $P$-value $=0.066$. This relative advantage of the $6 \mathrm{M}$ for the FOLFOX subgroup is driven by life expectancy (see Table S7 in supplementary material for a detailed subgroup survival analysis). While for the CAPOX regimen, life expectancy is better for the $3 \mathrm{M}$ arm by 0.07 years, patients treated with FOLFOX have a greater life expectancy in the $6 \mathrm{M}$ arm, by 0.22 years (interaction test, $P$-value $=0.106$ ). In the FOLFOX subgroup, the 3 $M$ arm still maintains a greater NMB than $6 \mathrm{M}$, with an incremental NMB of $£ 3229$ and a $77 \%$ probability of being cost-effective given a UK threshold of $£ 30,000 / Q A L Y$. Therefore, the $6 \mathrm{M}$ arm would not be considered cost-effective and the $3 \mathrm{M}$ treatment duration remains the optimal treatment strategy.

The interaction test of differences in incremental QALYs between the risk, gender and age subgroups showed no significant differences in incremental QALYs.

For each subgroup, the $3 \mathrm{M}$ strategy has the highest probability of being cost effective for a wide range of monetary values of a QALY (see Figure $\mathrm{S} 5$ in supplementary material for the cost-effectiveness acceptability curves). Only when a threshold higher than $£ 60,000$ / QALY is considered the $6 \mathrm{M}$ arm becomes the cost-effective strategy (with highest probability) for the FOLFOX subgroup.

\section{DISCUSSION}

The results of the economic evaluation show that the 3-month duration arm is clearly a cheaper intervention and the dominant strategy for chemotherapy treatment for high-risk stage II and stage III CRC. A short treatment duration significantly reduces costs by saving resources related to adjuvant chemotherapy and hospitalisations. The $3 \mathrm{M}$ arm also significantly improves patient quality of life during the treatment period, with no significant impact on overall survival, leading to an overall QALY gain for the $3 \mathrm{M}$ arm, albeit not statistically significant. The probabilistic analysis and exploration of cost-effectiveness acceptability showed little uncertainty in the economic results over a wide range of willingness to pay thresholds.

An exploration of four subgroup analyses indicates similar conclusions, with the $3 \mathrm{M}$ arm being the dominant or costeffective strategy given the UK $£ 30,000$ per QALY threshold. The type of chemotherapy received has the greatest subgroup impact on cost-effectiveness. The interaction between planned treatment and QALY differences between the two arms is statistically significant. In the analyses of the FOLFOX chemotherapy regimen, the $6 \mathrm{M}$ arm generated more QALYs than the $3 \mathrm{M}$ arm, primarily driven by the higher estimated disease free and overall survival, which was explored and discussed in the main study analyses. ${ }^{20}$ The quality of life difference between arms is in favour of the 3 month arm, yet the survival gains in the FOLFOX regimen are relevant over the 8 year follow-up period, resulting in an overall QALY gain. Nonetheless, at a decision threshold of $£ 30,000 / Q A L Y$ the NMB is larger for the $3 \mathrm{M}$ arm (incremental gain of $£ 3229$ per patient) and therefore the $6 \mathrm{M}$ duration would not be considered cost-effective from a UK perspective. ${ }^{21}$

Strengths: The 8 year patient follow-up allowed exploration of the cost-effectiveness outcomes over this long time period. The key cost differences occur within the first year after randomisation, where the $6 \mathrm{M}$ arm costs nearly double the $3 \mathrm{M}$ arm in chemotherapy and hospitalisations. Between years 1 to 8 there is little difference in hospitalisations between arms, yet over the 8year analysis period the total costs per patient are significantly different between arms, favouring the $3 \mathrm{M}$ arm. We would not expect longer-term differences in costs between arms. The followup also allows a thorough consideration of the QALY results which are driven by the timing of events. There are small differences in life expectancy which are subject to uncertainty in both the base case and subgroup analyses, yet, overall quality of life is consistently higher in the $3 \mathrm{M}$ arm in all analyses. The maximum advantage in quality of life is between 3 to 6 months post randomisation where the $3 \mathrm{M}$ arm have stopped treatment. The detrimental quality of life impact on the $6 \mathrm{M}$ arm remains due to the adverse event derived from chemotherapy. ${ }^{30}$

Limitations: Data present censoring given the minimum of $3-$ year follow up and the relatively high survival rates after 8 years (about 75\%). This aspect could be affecting the results to some extent. The cost and quality-adjusted survival methodology used, i.e., KMSA and partitioned survival analysis respectively, is chosen to reduce censoring-related biases.

This study was undertaken using the within-trial 8 years time horizon. The analysis shows that the key differences between arms in terms of costs and quality of life occur mainly within the first year, and beyond this point there are no significant differences between the two strategies. Nonetheless, extrapolation beyond the 8 years is unlikely to change the outcomes and conclusions unless we anticipate huge differences in survival.

\section{CONCLUSION}

This study found that compared with the traditional 6-month chemotherapy treatment period, a 3 months treatment strategy costs significantly less with no significant detrimental impact on patient outcomes (quality of life and survival) and therefore is found to dominate. Three months chemotherapy treatment for patients with stage III and high-risk stage II CRC is the optimal treatment strategy from an UK health-care perspective. Costeffectiveness is affected by the type of chemotherapy regimen used, however, the $3 \mathrm{M}$ strategy remains the optimal choice for both the CAPOX and FOLFOX regimens under a policy relevant willingness to pay thresholds.

The SCOT trial was the largest randomised study in adjuvant treatment of CRC to date, which showed that $3 \mathrm{M}$ treatment is noninferior to $6 \mathrm{M}$ treatment in the overall trial population. This economic evaluation undertaken alongside SCOT adds to the evidence showing 
SCOT: a comparison of cost-effectiveness from a large randomised phase...

that the $3 \mathrm{M}$ treatment strategy is not only cost-effective, but a dominant treatment strategy with little uncertainty in the costeffectiveness decision; thus providing the economic case that a $3 \mathrm{M}$ treatment strategy should be considered a new standard of care.

\section{ACKNOWLEDGEMENTS}

This work was supported by Medical Research Council (transferred to NETSCCEfficacy and Mechanism Evaluation) (Grant Ref: G0601705) and Cancer Research UK Core CTU Funding (Funding Ref: C6716/A9894). The Study Sponsor was NHS Greater Glasgow \& Clyde and University of Glasgow. Eudract Ref: 2007-003957-10; ISRCTN No.: 23516549 .

\section{AUTHORS CONTRIBUTIONS}

Dr José Robles-Zurita: Corresponding author, undertook health economic analysis, data analysis and manuscript writing. Dr Kathleen Anne Boyd: Led health economic analysis, data analysis, manuscript writing and review. Dr Andrew H Briggs: Grant Submission, study concept \& design, manuscript review. Dr Timothy Iveson: Study concept \& design, trial supervision, patient recruitment, manuscript review. Dr Rachel Kerr: Study concept \& design, trial supervision, and manuscript review. Dr Mark P Saunders: Trial supervision, patient recruitment and manuscript review. Jim Cassidy: Grant Submission, study concept \& design, trial supervision, patient recruitment, review of manuscript. Niels Henrik Hollander: Trial supervision, patient recruitment, review of manuscript. Josep Tabernero: Trial supervision, patient recruitment, review of manuscript. Eva Segelov: Trial supervision, review of manuscript. Bengt Glimelius: Trial supervision, patient recruitment, review of manuscript. Andrea Harkin: Grant Submission, data interpretation, manuscript writing and review. Karen Allan: Grant Submission, data collection, and manuscript review. John McQueen: Data collection, data interpretation, and manuscript review. Sarah Pearson: Data interpretation, and manuscript review. Ashita Waterston: Patient recruitment, review of manuscript. Louise Medley: Patient recruitment, review of manuscript. Charles Wilson: Patient recruitment, review of manuscript. Richard Ellis: Patient recruitment, review of manuscript. Sharadah Essapen: Patient recruitment, review of manuscript. Amandeep Dhadda: Patient recruitment, review of manuscript. Rob Hughes: Patient recruitment, review of manuscript. Stephen Falk: Patient recruitment, review of manuscript. Sherif Raouf: Patient recruitment, review of manuscript. Charlotte Rees: Patient recruitment, review of manuscript. Rene $\mathrm{K}$ Olesen: Patient recruitment, review of manuscript David Propper: Patient recruitment, review of manuscript. John Bridgewater: Patient recruitment, review of manuscript. Ashraf Azzabi: Patient recruitment, review of manuscript. David Farrugia: Patient recruitment, review of manuscript. Andrew Webb: Patient recruitment, review of manuscript. David Cunningham: Patient recruitment, review of manuscript. Tamas Hickish: Patient recruitment, review of manuscript. Andrew Weaver: Patient recruitment, review of manuscript. Simon Gollins: Patient recruitment, review of manuscript. Harpreet Wasan: Patient recruitment, review of manuscript. James Paul: Grant Submission, study concept \& design, data interpretation, data analysis, manuscript writing and review.

\section{ADDITIONAL INFORMATION}

Supplementary information is available for this paper at https://doi.org/10.1038/ s41416-018-0319-z.

Competing interests: T.I. reports Honoraria from Lilly Advisory Boards for Servier, Roche and Celgene Travel expenses from Bayer and Servier outside the submitted work. J.B. reports personal fees from Servier, Celgene and Merck, and non financial supports from Merck Sharpe and Dohme outside the submitted work. C.W. reports grants from sirtex-european symposium Nov 2016, outside the submitted work. D.C. reports grants from Amgen, AstraZeneca, Bayer, Celgene, Merrimack, Medlmmune, Merck Serono and Sanofi, outside the submitted work. J.C. reports grants from MRC and CRUK, and confirms that he is currently an employee with Celgene Corporation. K.A.B. reports grants from Medical Research Council during the conduct of the study. J.T. reports other from Amgen, Bayer, Boehringer Ingelheim, Celgene, Chugai, Lilly, MSD, Merck Serono, Novartis, Pfizer, Roche, Sanofi, Symphogen, Taiho and Takeda, outside the submitted work. A.W. is involved in research that is funded by drug companies that provide the drugs for the study. She has been co-investigator in trials funded by Roche that provide Capecitabine used in this study. B.G. sits on the Advisory Board for PledPharma AB.

Ethics committee approval: This study was approved by the West Glasgow Research Ethics Committee (version 1.1 of the protocol) on 21st January 2008 and all subsequent amendments approved by the Committee (where required). REC reference number: 07/S0703/136.

\section{REFERENCES}

1. CRUK. Bowel cancer statistics 2016 [http://www.cancerresearchuk.org/healthprofessional/cancer-statistics/statistics-by-cancer-type/bowel-cancer\#headingZero.

2. Laudicella, M., Walsh, B., Burns, E. \& Smith, P. C. Cost of care for cancer patients in England: Evidence from population-based patient-level data. Br. J. Cancer 114 1286-1292 (2016).

3. Sullivan, R. et al. Delivering affordable cancer care in high-income countries. Lancet Oncol. 12, 933-980 (2011).

4. Efficacy of adjuvant fluorouracil and folinic acid in colon cancer. International Multicentre Pooled Analysis of Colon Cancer Trials (IMPACT) investigators. Lancet 345, 939-944 (1995).

5. Haller D., Catalano P., Macdonald J., Mayer R., editors. Fluorouracil (FU), leucovorin (LV) and levamisole (LEV) adjuvant therapy for colon cancer: five-year final report of INT-0089. Proc Am Soc Clin Oncol; 1998.

6. O'Connell, M. J. et al. Prospectively randomized trial of postoperative adjuvant chemotherapy in patients with high-risk colon cancer. J. Clin. Oncol. 16, 295-300 (1998).

7. O'Connell, M. J. et al. Controlled trial of fluorouracil and low-dose leucovorin given for 6 months as postoperative adjuvant therapy for colon cancer. J. Clin. Oncol. 15, 246-250 (1997).

8. Wolmark, N. et al. The benefit of leucovorin-modulated fluorouracil as postoperative adjuvant therapy for primary colon cancer: results from National Surgical Adjuvant Breast and Bowel Project protocol C-03. J. Clin. Oncol. 11, 1879-1887 (1993).

9. Wolmark, N. et al. Clinical trial to assess the relative efficacy of fluorouracil and leucovorin, fluorouracil and levamisole, and fluorouracil, leucovorin, and levamisole in patients with Dukes' $B$ and $C$ carcinoma of the colon: results from National Surgical Adjuvant Breast and Bowel Project C-04. J. Clin. Oncol. 17, 3553-3559 (1999).

10. Zaniboni, A. Adjuvant chemotherapy in colorectal cancer with high-dose leucovorin and fluorouracil: impact on disease-free survival and overall survival. $J$. Clin. Oncol. 15, 2432-2441 (1997).

11. Haller, D. G. et al. Phase III study of fluorouracil, leucovorin, and levamisole in high-risk stage II and III colon cancer: final report of intergroup 0089. J. Clin. Oncol. 23, 8671-8678 (2005).

12. André, T. et al. Oxaliplatin, fluorouracil, and leucovorin as adjuvant treatment for colon cancer. New Engl. J. Med. 350, 2343-2351 (2004).

13. Haller, D. G. et al. Capecitabine plus oxaliplatin compared with fluorouracil and folinic acid as adjuvant therapy for stage III colon cancer. J. Clin. Oncol. 29, 1465-1471 (2011).

14. Kuebler, J. P. et al. Oxaliplatin combined with weekly bolus fluorouracil and leucovorin as surgical adjuvant chemotherapy for stage II and III colon cancer: results from NSABP C-07. J. Clin. Oncol. 25, 2198-2204 (2007).

15. Land, S. R. et al. Neurotoxicity from oxaliplatin combined with weekly bolus fluorouracil and leucovorin as surgical adjuvant chemotherapy for stage II and III colon cancer: NSABP C-07. J. Clin. Oncol. 25, 2205-2211 (2007).

16. Pachman, D. R. et al. Clinical course of oxaliplatin-induced neuropathy: results from the randomized phase III trial N08CB (alliance). J. Clin. Oncol. 33, 3416-3422 (2015).

17. Schrag, D. The price tag on progress-chemotherapy for colorectal cancer. New Engl. J. Med. 351, 317-319 (2004).

18. Aballéa, S. et al. Cost-effectiveness analysis of oxaliplatin compared with 5fluorouracil/leucovorin in adjuvant treatment of stage III colon cancer in the US. Cancer 109, 1082-1089 (2007).

19. Cassidy, J. et al. Pharmacoeconomic analysis of adjuvant oral capecitabine vs intravenous 5-FU/LV in Dukes' C colon cancer: the X-ACT trial. Br. J. Cancer 94 1122 (2006).

20. Iveson, T. J. et al. 3 versus 6 months of adjuvant oxaliplatin-fluoropyrimidine combination therapy for colorectal cancer (SCOT): an international, randomised, phase 3, non-inferiority trial. Lancet Oncol. 19, 562-578 (2018).

21. National Institute for Health and Care Excellence. Guide to the Methods of Technology Appraisal: 2013. 2013.

22. Petrou, S. \& Gray, A. Economic evaluation alongside randomised controlled trials: design, conduct, analysis, and reporting. BMJ 342, d1548 (2011).

23. Husereau, D. et al. Consolidated health economic evaluation reporting standards (CHEERS) - explanation and elaboration: a report of the ISPOR health economic evaluation publication guidelines good reporting practices task force. Value Health 16, 231-250 (2013).

24. Billingham, L. \& Abrams, K. Simultaneous analysis of quality of life and survival data. Stat. Methods Med. Res. 11, 25-48 (2002).

25. Dolan, P. Modeling valuations for EuroQol health states. Med. Care 35, 1095-1108 (1997).

26. Billingham, L., Abrams, K. \& Jones, D. Methods for the analysis of quality-of-life and survival data in health technology assessment. Health Technol. Assess. (Winch., Engl.). 3, 1 (1999). 
SCOT: a comparison of cost-effectiveness from a large randomised phase...

$\mathrm{J}$ Robles-Zurita et al.

1338

27. BNF British National Formulary 73 , Medicines Complete.

28. Information Services Division. NHS National Services Scotland. Scottish Health Service Costs 2016 [www.isdscotland.org/costs].

29. Lin D., Feuer E., Etzioni R., Wax Y. Estimating medical costs from incomplete follow-up data. Biometrics 53, 419-434 (1997).

30. Boyd, K. A. et al. Analysis of adverse events and quality of life data for an economic evaluation of adjuvant chemotherapy in colorectal cancer: when can we stop collecting? Trials 12(S1), A41 (2011).

31. Briggs, A. H., Wonderling, D. E. \& Mooney, C. Z. Pulling cost-effectiveness analysis up by its bootstraps: A non-parametric approach to confidence interval estimation. Health Econ. 6, 327-340 (1997).

32. Fenwick, E., Claxton, K. \& Sculpher, M. Representing uncertainty: the role of costeffectiveness acceptability curves. Health Econ. 10, 779-787 (2001). (c) (1) Open Access This article is licensed under a Creative Commons Attribution 4.0 International License, which permits use, sharing, adaptation, distribution and reproduction in any medium or format, as long as you give appropriate credit to the original author(s) and the source, provide a link to the Creative Commons license, and indicate if changes were made. The images or other third party material in this article are included in the article's Creative Commons license, unless indicated otherwise in a credit line to the material. If material is not included in the article's Creative Commons license and your intended use is not permitted by statutory regulation or exceeds the permitted use, you will need to obtain permission directly from the copyright holder. To view a copy of this license, visit http://creativecommons. org/licenses/by/4.0/

() The Author(s) 2018 